\title{
Coalitional Bargaining in Networks
}

\author{
Thành Nguyen* \\ This version: January 2014
}

\begin{abstract}
We analyze an infinite horizon, non-cooperative bargaining game with a general coalition structure. In each period an opportunity for a feasible coalition to form arises according to a stochastic process, and a randomly selected agent in the coalition makes a take-it-orleave-it offer to the other agents in the coalition. We introduce a new technique based on convex programming to characterize the stationary equilibrium of the game. We apply the framework to various settings, where the feasible coalitions are determined by an underlying trading network. We study the effect of the underlying network to the pattern of trade and show how an agent's payoff is related to his position in the network.
\end{abstract}

KEYWORDS: Network Games, Non-cooperative Bargaining, Coalition Formation

\section{Introduction}

In studying and modeling the economics of trade, economists have long recognized that barriers-institutional, physical, and otherwise-inhibit direct trade between some pairs of agents. In order to overcome such barriers to trade, agents naturally forge networks among themselves. As such, economists, beginning with Kantorovich [1960] and Koopmans [1949] ]1 have used network models to study economic environments with trade barriers. Inquiry into the effects of network structure on the equilibrium division of surplus has important implications for network design. Previous efforts towards modeling such environments, however,

*Email: nguye161@purdue.edu.

${ }^{1}$ The original version of Kantorovich [1960] was published in Russian in 1939. See Schrijver [2005] for details. 
have either abstracted away from non-cooperative bargaining or primarily focused on coalition network structures containing only pairs of agents. Our paper, by providing a more general framework that allows for nonuniform surplus functions and multilateral bargaining among agents, improves upon prior research into the effects of network structure on outcomes of trade. More specifically, our technique, based on convex programming, facilitates modeling various economic environments that cannot be represented by models with coalitions composed solely of pairs of agents.

An important first step in the literature is taken by Myerson [1977], who begins with the familiar notion of a transferable utility game $(N, V)$, where $N$ is the set of agents and $V$ is the characteristic function denoting the total surplus $V(S)$ for each coalition $S \subset N$. He uses a network structure to define the possible communication or cooperation that could take place. Specifically, cooperation among individuals is only possible when they are connected by an underlying network. This is a powerful framework that models a variety of economic environments. For example, in a supply chain, the characteristic function $V(S)$ represents the total surplus that all agents in $S$ together can generate by producing, delivering and consuming certain goods. In labor markets, $V(\{f\} \cup W)$ models the surplus created by a firm $f$ and a set of workers $W$. The risk-sharing market is another example of an environment in which network models can provide useful insights. Here agents holds risky assets, and $V(S)$ represents the gain of combining the assets own by agents in $S$ to reduce risk. In all of these examples, the underlying network can model various physical or institutional constraints that prevent trade or cooperation among certain groups of agents.

The main question addressed in Myerson [1977] is how the underlying coalition structure influences the manner in which surplus is split among its members. To answer this, Myerson extends the Shapley value, a well known concept in cooperative game theory, to a network setting and proves that if the sharing rules satisfy certain "fairness" and "efficiency" properties, there is a unique way that the agents should share the surplus. In spite of its elegance, this approach is not entirely satisfactory, because it abstracts away the incentive of individuals in the bargaining process. On the other hand, models of non-cooperative bargaining 
games in which agents' payoffs are determined by the outcome of the bargaining process are generally too complex in a network setting and are, thus, analytically intractable.

More recently, Manea [2011] presents a tractable non-cooperative network bargaining model. In his paper, the coalition structure consists of pairs of agents who are connected by a network, and the surplus generated by such pairs is assumed to be uniform. Even with this simplification, the model provides new insights into the influence of the underlying network on agents' bargaining power. In particular, in this model, trade opportunities are defined by a heterogeneous network, in which an agent's bargaining power depends not only on her rate of making an offer as in the basic bargaining game of Rubinstein [1982], but also on the bargaining power of her neighbors. The bargaining power of these agents also depends on their neighbors, which gives rise to a network effect. Manea [2011] develops a clever algorithm to compute the unique equilibrium payoff and provides a characterization of the payoff based on a statistic of the underlying network called shortage-ratios.

However, there are various economic environments that cannot be captured by coalition structures containing only pairs of agents. For example, trade in a supply chain often involves middlemen; portfolios in a risk-sharing market are usually combinations of more than two assets. It is not clear whether Manea's analysis can be extended to such settings.

The contribution of our paper is to extend Manea [2011] to a more general setting that allows multilateral bargaining and a nonuniform surplus function. In order to do that, we develop a new technique based on convex programming to characterize the equilibrium payoffs.

Specifically, the bargaining model we consider in this paper is the combination of Myerson [1977] and Manea [2011]. As in Mverson [1977], we start with a transferable utility game $(N, V)$ and let a general set family $\mathcal{F} \subset 2^{N}$ to be the set of feasible coalitions. Following Manea [2011], the bargaining is defined as an infinite-horizon repeated game. In each period, one coalition is selected from $\mathcal{F}$ according to a probability distribution, and then an agent in the coalition is chosen at random to be the proposer. The proposer can decline to propose or suggest a division of the coalition surplus to its members. If the proposer declines 
to propose, or at least one member of the coalition objects to the suggested share, the game continues to the next stage where another coalition is selected. Otherwise, agents in the coalition exit the game with their proposed division, and are replaced by clones. The game repeats 2

We show that stationary equilibria exist; furthermore, the payoff of any stationary equilibrium is the unique solution of a quadratic convex program. A constraint of this program corresponds to a feasible coalition. If a constraint does not bind in the optimal solution, then the corresponding coalition will never form at equilibrium. Prior bargaining literature has demonstrated that a coalition in which agents never reach an agreement in equilibrium cannot be used as a credible outside option for these agents when bargaining with others. Therefore, they are redundant and should not influence bargaining power. We show that the primal-dual relationship of the convex program characterization gives a natural interpretation for such a relationship between bargaining power and credible outside options.

We apply the convex program characterization and its dual program to several network games in Section 4. Depending on the application, we define the set of feasible coalitions and their surplus based on an underlying network. We study the influence of the underlying network to the set of coalitions formed at equilibrium as well as the bargaining power of the agents. Specifically, in Section 4.1, we study a market structure that involves middlemen, and compare it with the pairwise trading model in the seller-buyer networks of Manea [2011]. We extend this model to a general network in Section 4.2, where each feasible coalition is a path connecting a buyer and a seller. Here we prove an equivalence between the outcome of the bargaining game and an associated traffic routing game. This relationship highlights the connection between an agent's bargaining power and her degree of centrality in the network. Finally, in Section 4.3, we consider a game of cooperation in overlapping communities. We show that in this game a hierarchical structure among the agents emerges endogenously, and an agent at the intersection of communities has high bargaining power, which partly describes the social structural holes studied in Burt [1992]. Section 5] concludes, and the Appendix

\footnotetext{
${ }^{2}$ In Section 2 we introduce the precise model and discuss its interpretation in detail.
} 
contains the proofs.

\subsection{Other Related Literature}

Our paper is closely related to the literature on non-cooperative bargaining in dynamic markets studied in Rubinstein and Wolinsky [1985], Gale [1987], and more recently in Manea [2011]. Similar to these papers, we make the assumption that agents exit the market after a successful negotiation and are replaced by clones. This assumption allows us to focus on the steady-states of an on-going economy and, thus, reduces the complexity of the dynamic game substantially 3 However, unlike these papers, a coalition in our model can contain more than two agents.

Similar to our work, a recent paper of Siedlarek [2012] studies coalition structures beyond pairs of agents. However, Siedlarek [2012] uses a fixed point theorem to prove the existence of the equilibrium. Our characterization, based on a convex program, is more tractable for comparative analysis. Furthermore, Siedlarek [2012] imposes a new restriction: all of the coalitions contain one special node, corresponding to a single seller in the network.

Rather than generalizing the structure of the coalition, other authors explicitly model the assets being traded in order to extend pairwise bargaining models to trading environments with middlemen. Examples of papers that use this approach include Rubinstein and Wolinsky [1987], Wong and Wright [2014], Gofman [2011], Nguyen [2013], Manea [2013c] and Condorelli and Galeotti [2014]. In these models, the bargaining process depends on whether or not an agent holds an asset, and even though the bargaining occurs between pairs of agents, the overall trade involves multiple agents, because the assets are ultimately transferred through the entire network. However, to analyze these complex environments further restrictions are imposed. For example,Wong and Wright [2014], Gofman [2011], Manea [2013c] and Condorelli and Galeotti 2014] assume that there is only one unit of indivisible good in the market, while Rubinstein and Wolinsky [1987] and Nguyen [2013] consider large economies consisting of infinitely many agents and

\footnotetext{
${ }^{3}$ For example, it has been shown in Abreu and Manea [2012a, b] that without the replacement assumption, there are multiple equilibria, and no general characterization of these equilibria is known.
} 
assets. In particular, Nguven [2013] shows that the convex program technique developed in this paper can be extended to analyze one such environment.

More broadly, our paper contributes to the large and expanding literature on game theoretical network models. For an extensive review, see, for example, Jackson [2008]. One central question of this literature is how the network structure influences agents' payoffs. A number of answers and approaches to this question have been provided by, for example, Ballester et al. [2006] and Golub and Elliott [2013]. These papers, however, assume specific forms of externalities and complementarities among the agents and, furthermore, do not allow for transfers between agents. Thus, the channels through which the network structure affects agents' payoffs in these approaches are quite different from those in our work. Our paper also studies the formation of trade and coalition over a network structure, and thus has a connection with the literature on network formation (Jackson and Wolinsky 1996], Kranton and Minehart [2001], Bala and Goyal [2002], Goyal and Vega-Redondo [2007] and Elliott [2014]). Similar to these papers, our result provides a characterization on the coalitions and links that form at equilibrium. However, our approach is different and based on a dynamic, non-cooperative bargaining game. Thus, for example, we are able to investigate the impact of the discount rate on network formation.

\section{Coalitional Bargaining Framework}

\subsection{The Model}

Suppose a set of $n$ agents, $N=\{1, \ldots, n\}$, and let $\mathcal{F} \subset 2^{N}$ be the set of feasible coalitions. Denote by $V(S) \geq 0$ the non-negative surplus of coalition $S \in \mathcal{F}$. Consider the following infinite horizon bargaining game. At the beginning of each period $t=1,2, \ldots, \infty$, a coalition $S \in \mathcal{F}$ and one of the agents, $i \in S$, is selected according to a distribution satisfying the 
following product form 4

$$
\operatorname{Pr}(S, i)=\alpha_{i} \cdot P(S), \text { where } \alpha_{i}, P(S)>0
$$

Agent $i$ becomes the proposer. The proposer can potentially decline to make any offer, in which case then the game moves to the next period. On the other hand, if $i$ decides to make an offer, then she suggests a division of $V(S)$ to agents in $S-\{i\}$ sequentially according to a fixed priority order. If one of the agents in $S-\{i\}$ rejects the offer, period $t$ ends. If all agents agree, all agents in $S$ exit the game with the shares they agreed on, and are replaced by clones.

The game repeats in period $t+1$. Let $\delta_{i} \in(0,1)$ be the discount factor of agent $i$ for each period. The game is denoted by

$$
\Gamma(N, \mathcal{F}, V(.), P(.), \vec{\alpha}, \vec{\delta}) .
$$

\subsection{Stationary Equilibrium}

In this paper, we focus on stationary equilibrium: a subgame perfect equilibrium, in which each player's strategy depends only on the play of the game in the current period and not on the history of the game from the past periods.

Each period of the game consists of a finite number of sub-periods, which correspond to the sequential moves of the players. In a given period, let $h_{\tau}$ be the history of the game from the time when the period starts until the beginning of sub-period $\tau$.

Assume at sub-period $\tau$ agent $i$ makes an offer to agent $j \in S$, then a pure strategy of agent $i$ at this sub-period is a function $\sigma_{i, \tau}$ that maps $\left(h_{\tau}, j\right)$ to an $m \in \mathbb{R}$ or to $\{\varnothing\}$. Here $m$ represents the transfer that $i$ offers to $j ; \varnothing$ corresponds to $i$ 's refusal to make any offer to $j$. On the other hand, agent $j$ 's pure strategy maps the history $h_{\tau}$ and the transfer $m$ offered by $i$ to a binary decision: Accept or Reject.

Our solution concept allows mixed strategies, which are distributions over pure strategies.

\footnotetext{
${ }^{4}$ The product form of this probability distribution is an important assumption for our characterization. The interpretation and examples for such assumptions are given in Section 2.3 .
} 


\subsection{Interpretations and Applications of the Model}

Our bargaining game models a large dynamic market, in which there is a finite set of agents' types, and each type consists of a large number of agents. Labor markets and asset markets are examples of such a model. These markets are not static, because labor and assets constantly arrive to and exit the marketplace. As in Rubinstein and Wolinsky [1985], we assume that after reaching an agreement, the agents involved in the negotiation exit the market and are replaced by clones. This assumption represents a steady-state of a dynamic market, where the arrival flow of newly born agents matches with the exiting flow for each type 5

The product form given in (11) captures a wide range of applications. One example is a labor market. Specifically, let $i \in N=\{1, \ldots, n\}$ represent a type of worker with similar skills and demographic information. For each type $i$, there is a continuum of workers with mass $\gamma_{i}>0$. Different types of jobs are represented by the set family $\mathcal{F}$ and the surplus function $V($.$) . Each period, a mass \gamma$ of jobs with type $S \in \mathcal{F}$ arrive with probability $p_{S}$. We assume $\gamma<\min _{i} \gamma_{i}$. Workers apply to these jobs, and for every $i \in S$ a mass $\gamma$ of workers of type $i$ is selected at random. For each job, among $|S|$ workers, one of them is chosen at random and proposes to other $|S|-1$ workers a way to share the surplus $V(S)$. Our model allows agents of different types to have different chance to be the proposer. Namely, given $\vec{\beta}>0$, let the probability that the agent $i$ becomes the proposer for the coalition $S \ni i$ be $\frac{\beta_{i}}{\sum_{j \in S} \beta_{j}}$. Thus, given $S$ and $i \in S$ the probability that a given worker of type $i$ is selected to be the proposer for coalition $S$ is

$$
\operatorname{Pr}(S, i)=p_{S} \cdot \frac{\gamma}{\gamma_{i}} \cdot \frac{\beta_{i}}{\sum_{j \in S} \beta_{j}}=\frac{\gamma \beta_{i}}{\gamma_{i}} \cdot \frac{p_{S}}{\sum_{j \in S} \beta_{j}},
$$

which satisfies the product form given in (1).

Our model can also be applied to analyze the effects of network structure in risk-sharing markets. Here each agent owns a risky asset. Let $N=\{1, . ., n\}$ represent the types of risky

\footnotetext{
${ }^{5}$ Such a steady-state can be made endogenous with proper parameters on the rate of newly born agents and their outside option. One can use the frameworks similar to Gale [1987], Manea [2013a, b] to endogenize the steady-state assumption in this setting.
} 
assets. Each $S \subset N$ corresponds to a portfolio that consists of $|S|$ assets of different types. $V(S)$ represents the benefit of risk-pooling by combining these assets into one portfolio 6 Assume that in a steady-state, there is a mass $\gamma_{i}$ of assets of type $i \in N$ in the market. Similar to the labor market above, in every period, with $p_{S}(\vec{\gamma})$ probability 7 there is a demand for a mass $\gamma<\min _{i} \gamma_{i}$ of portfolios $S$. For each $i \in S$, a mass $\gamma$ of agents owning asset $i$ are selected at random. These agents will bargain on how to split $V(S)$. We again assume that condition on being selected in a coalition $S$, the probability that a particular agent $i$ becomes the proposer is $\frac{\beta_{i}}{\sum_{j \in S} \beta_{j}}$. Thus, the probability that a given agent $i$ becomes the proposer for the coalition $S \ni i$ is

$$
\operatorname{Pr}(S, i)=\frac{\gamma}{\gamma_{i}} p_{S}(\vec{\gamma}) \cdot \frac{\beta_{i}}{\sum_{j \in S} \beta_{j}}=\frac{\gamma \beta_{i}}{\gamma_{i}} \cdot \frac{p_{S}(\vec{\gamma})}{\sum_{j \in S} \beta_{j}},
$$

which also follows the product form given in (1).

Notice that both labor and asset markets are dynamic, because when a coalition of workers forms or a portfolio is generated, these workers and assets exit the search market. At the same time, new labor and assets are arriving to the market.

\section{Equilibrium Characterization}

We give the following characterization (Theorem 1) of the stationary equilibrium payoffs of the bargaining game, $\Gamma(N, \mathcal{F}, V(),. P(),. \vec{\alpha}, \vec{\delta})$, defined above. This result also allows us to identify the set of coalitions that are formed in equilibrium. In Theorem 2, we then analyze the equilibrium payoff when the discount rates approach 1.

THEOREM 1 Given the game $\Gamma(N, \mathcal{F}, V(),. P(),. \vec{\alpha}, \vec{\delta})$, the payoff of any stationary equilibrium is the unique optimal solution of the following convex program on the variables $u_{i}, z_{S}$.

$$
\begin{array}{ll}
\text { minimize: } & \sum_{i \in N} \frac{\delta_{i}\left(1-\delta_{i}\right)}{\alpha_{i}} u_{i}^{2}+\sum_{S \in \mathcal{F}} P(S) z_{S}^{2} \\
\text { subject to: } & \sum_{i \in S} \delta_{i} u_{i}+z_{S} \geq V(S) \quad \forall S \in \mathcal{F} .
\end{array}
$$

${ }^{6}$ See for example, Csóka et al. [2009] for such a model of valuation.

${ }^{7}$ Such $p_{S}($.$) is usually called matching function in the literature on markets with search frictions.$ 
Furthermore, let $u^{A}, z^{A}$ be the optimal solution of (A), then a stationary equilibrium is characterized as follows. When a coalition $S$ is selected and $i \in S$ is the proposer, then with probability 1:

- if $\sum_{j \in S} \delta_{j} u_{j}^{A}<V(S)$, $i$ offers $\delta_{j} u_{j}^{A}$ to every $j \in S$ and $j$ accepts,

- if $\sum_{j \in S} \delta_{j} u_{j}^{A}>V(S)$, either $i$ refuses to make an offer or least one agent $j \in S$ rejects.

Remark. We do not need the constraints $u_{i} \geq 0$ and $z_{S} \geq 0$ in the program (A) because in the optimal solution, $u_{i}, z_{S}$ are always non-negative. This is true because otherwise once can replace them by 0 to obtain a better solution. Also notice that Theorem 1 does not imply uniqueness of equilibrium strategies. For example, if $\sum_{j \in S} \delta_{j} u_{j}^{A}=V(S)$ then all (randomized) combinations of accepting and rejecting are in equilibrium. However, for our purpose, expected payoff of the agents and the pattern of trade, characterized by the coalitions formed at equilibrium, are uniquely determined.

The key technical difference between Manea's approach and ours is the following. To show the existence of a stationary equilibrium Manea [2011] used a fixed point theorem. On the other hand, we interpret the recursive formulation for $\vec{u}$ to be a stationary equilibrium payoff as the first-order condition of a convex program. However, because there are several such programs, we will need to carefully select one such that its complementary-slackness conditions can be also derived from the recursive equilibrium condition. This is a new method to characterize the equilibrium of a dynamic game. Compared with a characterization based on fixed point theorems, our convex program is more tractable and easier to compute. In particular, in Appendix $\mathrm{C}$ we show a new way to compute the equilibrium outcome of the model in Manea [2011].

Proof. Proof of Theorem 1: Assume a stationary equilibrium exists. Let $u_{i}$ be the expected payoff of agent $i$ in any subgame of such equilibrium. Assume at the beginning of the period, coalition $S$ is selected and $i$ is chosen as the proposer. We consider three cases: 
First, when $\sum_{j \in S} \delta_{j} u_{j}>V(S)$ then if $S$ is formed, there will be at least one agent $j$ that gets less than her continuation payoff, $\delta_{j} u_{j}$. That agent would be better off rejecting the offer (or declining to propose if she is the proposer). Therefore, with probability 1 this coalition will not be formed.

Second, if $\sum_{j \in S} \delta_{j} u_{j}<V(S)$, we will show that the only subgame perfect stationary strategy is that $i$ offers $\delta_{j} u_{j}$ to agent $j \in S-\{i\}$ and $j$ accepts with probability 1 . We prove this with an argument based on backward induction on the game tree representing the sequential offers made by agent $i$. Let $k=|S|-1$, and without loss of generality, assume the agents in $S \backslash\{i\}$ are indexed from 1 to $k$ according to the order in which $i$ proposes.

Consider the last agent, $k$. Notice that the game only reaches this point if, in all previous subperiods, $i$ did not decline and all other agents have accepted $i$ 's offer. Let $x_{1}, x_{2}, \ldots, x_{k-1}$ be the offers in the corresponding subperiod. We claim that if $V(S)-\left(x_{1}+\cdots+x_{k-1}\right)>$ $\delta_{k} u_{k}+\delta_{i} u_{i}$, then $i$ will propose $\delta_{k} u_{k}$ to $k$ and $k$ accepts with probability 1 .

This is true because this is the last subperiod; $j$ will accept an offer larger than her continuation payoff $\left(\delta_{k} u_{k}\right)$ and reject any offer less than that. However, any offer $x>\delta_{j} u_{j}$ cannot be an equilibrium, because $i$ can deviate to an offer, $x^{\prime}$, such that $x>x^{\prime}>\delta_{j} u_{j}$ and obtain a better payoff.

Consider the previous subperiod when $i$ proposes to $k-1$. We now claim that if $V(S)-$ $\left(x_{1}+\cdots+x_{k-2}\right)>\delta_{k-1} u_{k-1}+\delta_{k} u_{k}+\delta_{i} u_{i}$, then $i$ will propose $\delta_{k-1} u_{k-1}$ to $k-1$, and $k-1$ accepts with probability 1 . This is true for the following reason. Consider a small $\epsilon>0$, such that $\epsilon<V(S)-\left(x_{1}+\cdots+x_{k-2}+\delta_{k-1} u_{k-1}+\delta_{k} u_{k}+\delta_{i} u_{i}\right)$. If $i$ 's offer to $k-1$ is $x_{k-1}=\delta_{k-1} u_{k-1}+\epsilon$, then

$$
V(S)-\left(x_{1}+\cdots+x_{k-1}\right)>\delta_{k} u_{k}+\delta_{i} u_{i}
$$

Because of the previous argument, agent $k-1$ knows that $i$ 's offer to $k$ in the next subperiod will be accepted with probability 1 . Therefore, if $k-1$ accepts this offer, her payoff will be $\delta_{k-1} u_{k-1}+\epsilon$, which is strictly greater than her continuation payoff. Thus, $k-1$ accepts this offer with probability 1 . But again, $i$ can always deviate from this strategy by, for example, 
making an offer of $\delta_{k-1} u_{k-1}+\epsilon / 2$. Thus, the only equilibrium strategy at this subperiod is for $i$ to propose $x_{k-1}=\delta_{k-1} u_{k-1}$ to $k-1$, and $k-1$ accepts this offer with probability 1 .

Apply the same argument until we reach the first subperiod. At this step, we obtain that if $V(S)>\delta_{1} u_{1}+\cdots+\delta_{k} u_{k}+\delta_{i} u_{i}$, then the only subgame perfect equilibrium is for $i$ to offer $\delta_{j} u_{j}$ to every agent $j \in S-\{i\}$, and $j$ accepts with probability 1 , which is what we need to show. Now, in this case, agent $i$ ' payoff is $V(S)-\sum_{j \in S, j \neq i} \delta_{j} u_{j}$.

Finally, for the third case: if $\sum_{j \in S} \delta_{j} u_{j}=V(S)$, then there are multiple equilibrium strategies. However, regardless of the strategy, each agent $j$ gets a payoff of $\delta_{j} u_{j}$.

From the argument above, we have the following. When $S$ is chosen, and $i$ is selected to propose, $i$ 's payoff is

$$
\max \left\{V(S)-\sum_{j \in S, j \neq i} \delta_{j} u_{j}, \delta_{i} u_{i}\right\} .
$$

On the other hand, all other agents get their (discounted) continuation payoff.

We introduce the following notation

$$
z_{S}=\max \left\{V(S)-\sum_{k \in S} \delta_{k} u_{k}, 0\right\}
$$

to indicate whether a coalition $S$ will be formed. Namely, if $z_{S}>0$ the gain of the coalition $S$ is large enough, and $S$ will be formed. This notation also simplifies the payoff formula in (2); in particular, it can be written as $\max \left\{V(S)-\sum_{j \in S, j \neq i} \delta_{j} u_{j}, \delta_{i} u_{i}\right\}=\delta u_{i}+z_{S}$.

Now, the probability of $i$ being a proposer for coalition $S$ is $\alpha_{i} \cdot P(S)$. Therefore, agent $i$ 's ex-ante payoff in any subgame is

$$
u_{i}=\sum_{S \in \mathcal{F}: i \in S} \alpha_{i} \cdot P(S) \cdot\left(\delta_{i} u_{i}+z_{S}\right)+\left(1-\sum_{S \in \mathcal{F}: i \in S} \alpha_{i} \cdot P(S)\right) \delta_{i} u_{i} .
$$

This is equivalent to $u_{i}=\delta_{i} u_{i}+\sum_{S \in \mathcal{F}: i \in S} \alpha_{i} \cdot P(S) \cdot z_{S}$, and thus,

$$
\left(1-\delta_{i}\right) u_{i}=\alpha_{i} \cdot \sum_{S \in \mathcal{F}: i \in S} P(S) \cdot z_{S}
$$

We will show that the $\vec{u}$ which satisfies the conditions above is the unique solution of the 
following optimization program 8

$$
\begin{aligned}
\text { minimize: } & \sum_{i \in N} \frac{\delta_{i}\left(1-\delta_{i}\right)}{\alpha_{i}} u_{i}^{2}+\sum_{S \in \mathcal{F}} P(S) z_{S}^{2} \\
\text { subject to: } & \sum_{i \in S} \delta_{i} u_{i}+z_{S} \geq V(S) \quad \forall S \in \mathcal{F} .
\end{aligned}
$$

To prove the theorem, given a stationary equilibrium payoff $u$, let $z$ be defined as in (3), and let

$$
\lambda_{S}=2 P(S) z_{S}
$$

Consider $\lambda_{S}$ as the dual variable corresponding to the constraint $\sum_{i \in S} \delta_{i} u_{j}+z_{S} \geq V(S)$ of (A). We now show that the variables $x, z$ and $\lambda$ satisfy both the first order and the complementary slackness conditions of (A), which shows that $(x, z)$ is the unique solution of (A).

The first order conditions are

$$
2 \frac{\delta_{i}\left(1-\delta_{i}\right)}{\alpha_{i}} u_{i}=\sum_{S: i \in S} \delta_{i} \lambda_{S} \quad \text { and } \quad 2 P(S) z_{S}=\lambda_{S} .
$$

Both of these are satisfied because of the definition of $\lambda_{S}$ and (44).

Furthermore, the complementary slackness condition also follows. Namely, if $\sum_{i \in S} \delta_{i} u_{i}+$ $z_{S}>V(S)$, then $z_{S}>V(S)-\sum_{i \in S} \delta_{i} u_{i}$, and because of (3), $z_{S}=0$, which means the dual variable $\lambda_{S}=0$. Moreover, if $\lambda_{S}>0$, then $z_{S}>0$, therefore, $z_{S}=V(S)-\sum_{i \in S} \delta_{i} u_{i}$.

\subsection{When Agents Are Patient}

In order to isolate the effect of the coalitional structure on agents' bargaining power, in some of our applications, we focus on the case where agents are symmetric in terms of the discount factor. Namely, we assume $\delta_{i}=\delta$ for all $i$. We denote this game as $\Gamma^{\delta}$. According

\footnotetext{
${ }^{8}$ Intuitively, equation (4) can be seen as a duality condition of an optimization problem, where $z_{S}$ is the dual variable for a constraint $S$. However, $z_{S}$ also appears in (3). Hence, we have a problem where $z_{S}$ appears in both a primal and a dual program. To resolve this, for every $S \in \mathcal{F}$ we add a term $\operatorname{const}_{z} \cdot z_{S}^{2}$ to the objective function of a convex program. The first order condition corresponding to the variable $z_{S}$ represents a relation between $z_{S}$ and the dual variable of the constraint $\sum_{i \in S} \delta_{i} u_{i}+z_{S} \geq V(S)$. By adjusting these constants in the right way, we obtain this program.
} 
to Theorem 1, and by multiplying the objective of (A) by $\frac{1}{\delta(1-\delta)}$, we obtain the following program that characterizes the unique stationary equilibrium payoff:

$$
\begin{aligned}
& \text { minimize } \sum_{i \in N} \frac{1}{\alpha_{i}} u_{i}^{2}+\frac{1}{\delta(1-\delta)} \sum_{S \in \mathcal{F}} P(S) z_{S}^{2} \\
& \text { subject to } \sum_{j \in S} \delta u_{j}+z_{S} \geq V(S) \quad \forall S \in \mathcal{F} .
\end{aligned}
$$

We are interested in the outcome of $\Gamma^{\delta}$ as $\delta$ approaches 1 . When $\delta$ approaches 1 , the coefficient of $z_{S}$ in (A') goes to $\infty$. We will prove that in the optimal solution of (却), $z_{S}$ approaches 0 . This is true because otherwise, the objective function $\sum_{i \in N} \frac{1}{\alpha_{i}} u_{i}^{2}+\frac{1}{\delta(1-\delta)} \sum_{S \in \mathcal{F}} P(S) z_{S}^{2}$ can be arbitrarily large, which contradicts the fact that this is the minimum solution of (A'). Therefore, intuitively at the limit, one can replace $\delta=1$, and $z_{S}=0$ in (A') to obtain the following program.

$$
\begin{aligned}
& \text { minimize } \sum_{i \in N} \frac{1}{\alpha_{i}} u_{i}^{2} \\
& \text { subject to } \sum_{j \in S} u_{j} \geq V(S) \quad \forall S \in \mathcal{F} .
\end{aligned}
$$

The following theorem establishes convergence of $\Gamma^{\delta}$ as $\delta \rightarrow 1$.

THEOREM 2 Let $u^{* \delta}$ be the outcome of $\Gamma^{\delta}$ (optimal solution of (A1)) and $u^{*}$ be the solution of (B) , then $\lim _{\delta \rightarrow 1} u^{* \delta}=u^{*}$. Furthermore, there exists $0<\delta_{0}<1$ such that if a set $S \in \mathcal{F}$ satisfies $\sum_{i \in S} u_{i}^{*}>V(S)$, then the coalition $S$ will never be formed in any equilibria of the game $\Gamma^{\delta}$ for all $\delta \geq \delta_{0}$.

Proof. See Appendix A.1.

One important observation from the the characterization above is that $P(S)$ does not influence the limit solution as $\delta$ goes to 1 . The solution of (B) only depends on the set of feasible coalitions, $\mathcal{F}, \vec{\alpha}$, and the surplus function, $V($.$) . To see the intuition for this result,$ consider the following situation. When the discount rate $\delta$ is close to 1 , agents are patient and willing to wait for the best trading opportunities. Thus, for example, as long as $P(S)>0$ and the surplus $V(S)$ is large enough, agents will ignore some other coalitions to wait for $S$ to 
emerge. However, $\alpha_{i}$ corresponds to the relative frequency with which an agent $i$ is selected as a proposer compared with other agents; therefore, it influences the limit solution in (B).

\subsection{Interpretation of the Duals}

Let $u^{*}$ is the unique optimal solution of $(\underline{B})$, in the rest of the paper, we use $u_{i}^{*}$ as the index of bargaining power for agent $i$.

Consider the following primal-dual relation.

LEMMA $3.1 u^{*}$ is the optimal solution of (B) if and only if $u^{*}$ is feasible and there exist $\lambda_{S} \geq 0$ for all $S \in \mathcal{F}$ such that

$$
\begin{gathered}
u_{i}^{*}=\alpha_{i} \cdot \sum_{S \in \mathcal{F}: i \in S} \lambda_{S} \forall i \in N, \\
\lambda_{S}\left(\sum_{i \in S} u_{i}^{*}-V(S)\right)=0 .
\end{gathered}
$$

Lemma 3.1 can be interpreted as a duality between bargaining power and credible outside options. In particular, we can interpret the dual $\lambda_{S}$ as a measure of how credible the coalition $S$ is. Namely, when the constraint $S$ slacks, it corresponds to a coalition that will never be formed at equilibrium. In this case, $S$ cannot be considered to be a credible outside option and because of the duality result above, $\lambda_{S}=0$.

Now, also according to Lemma 3.1, $u_{i}^{*}=\alpha_{i} \cdot \sum_{S \in \mathcal{F}: i \in S} \lambda_{S} \forall i \in N$. Thus, for a noncredible outside option $S, \lambda_{S}=0$ and it does not affect an agent's bargaining power. In other words, the above primal-dual connection in our setting can be seen as a qualitative relation between bargaining power and credibility of outside options.

Finally, for the applications in the coming sections, we will need the following technical lemma.

LEMMA 3.2 If $u^{* \delta}$ is the optimal solution of (A), then there exist $\lambda_{S}^{\delta} \geq 0$ for all $S \in \mathcal{F}$ such that $\lambda_{S}^{\delta}=0$ for every $S$, of which the corresponding constraint in (A) does not bind and

$$
u_{i}^{* \delta}=\alpha_{i} \cdot \sum_{S \in \mathcal{F}: i \in S} \lambda_{S}^{\delta} \forall i \in N
$$


Both Lemma 3.1 and 3.2 follow from the Karush-Kuhn-Tucker conditions. The proof is standard and omitted. See, for example, Rockafellar [1970].

\section{Applications}

In this section we apply our characterization of the coalitional bargaining to several settings. To focus on the structure of the network, we assume $\delta_{i}=\delta$ and $\alpha_{i}=1$ for all $i$.

We start in Section 4.1 with an illustration of a market with two middlemen that connect different subsets of sellers and buyers. We study the equilibrium outcome under this market structure and compare it with the model of a market without middlemen studied in Manea [2011]. In Section 4.2, we extend that example to a general trading network. Finally, in Section 4.3, we consider a game of cooperation over a network.

\subsection{Trading with Middlemen}

We next consider a network that models a trading scenario involving middlemen. We will consider a simple such network to highlight some of the qualitative differences with the model of trade without middlemen studied in Manea [2011].

To capture middlemen, we consider the following network. Let $G$ be a "tripartite" network the nodes of which are partitioned into three classes: $S$ (sellers), $M$ (middlemen) and $B$ (buyers). We assume that the links of $G$ only connect nodes between $S-M$ and between $M-B$. To model the trading surplus, we assume the set of feasible coalitions to be $\mathcal{F}=$ $\{(s, m, b) \in S \times M \times B$ s.t $(s, m) ;(m, b)$ are both links in $G\}$. We further assume that the surplus of each coalition is 1 .

In the model of Manea [2011], the set of feasible coalitions consists of pairs of agents, and the surplus of each coalition is 1 . When the underlying network is bipartite, Manea showed that as the discount rate $\delta$ approaches 1, the market is segmented into several submarkets, each of which is a connected component of the sub-network formed by pairs of agents that trade with positive probability. Furthermore, in each sub-market, the payoffs of 
sellers (buyers) are identical. Namely, if a sub-market consists of a set of sellers $S$ and a set of buyers $B$, then the payoffs of a buyer and a seller are $\frac{|S|}{|S|+|B|}$ and $\frac{|B|}{|S|+|B|}$, respectively 9

To illustrate the differences with Manea [2011], consider the following network $G$ consisting of two middlemen 1 and 2. Middlemen $i, i \in\{1,2\}$ is connected to the set of buyers $B_{i}$ and the set of sellers $S_{i}$. We assume $B_{1}$ and $B_{2}$ are disjoint, but $S_{1}$ and $S_{2}$ are not. There are $\left|S_{1}\right|+\left|S_{2}\right|-|S|>0$ sellers that connect to both middlemen. See Figure 1 for a graphical representation of this network.

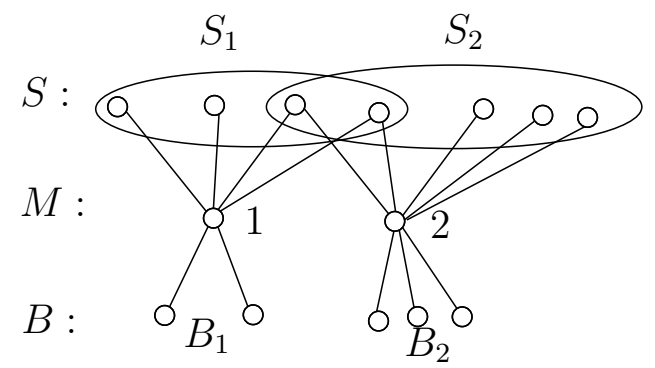

Figure 1: Seller buyer network and middlemen

In Theorem [3, we provide a comparative analysis on how the network influences the segmentation of the market. Specifically, under what conditions do the sellers who have access to both middlemen trade exclusively with only one of them? Under what conditions will they trade with both? The first case gives rise to a segmented market, while the latter results in a connected one. One would intuitively suspect that the extent of market segmentation depends on the number of sellers that have access to both middlemen, $\left|S_{1}\right|+\left|S_{2}\right|-|S|$. This is because at one extreme, if there are no such sellers, the market is obviously segmented. At the other extreme, when all sellers are connected to both middlemen, they would trade with both of them. Our analysis below, however, shows that the number sellers that have access to both the middlemen alone is not enough to determine if the market is segmented. The condition depends on both $\left|S_{1}\right|$ and $\left|S_{2}\right|$.

Another interesting property of the outcome for this network is that when the market is not segmented, the two groups of buyers will have different payoffs, 10 but all sellers obtain

\footnotetext{
${ }^{9}$ We provide an alternative proof, based on our convex program, of this result in Appendix C

${ }^{10}$ This property is in sharp contrast to Manea 2011].
} 
the same utility, even though some sellers are only connected to one middleman. Moreover, adding more links between sellers and middlemen will not further influence the outcome of the game. This illustrates a "phase transition" phenomenon, or "robust-yet-fragile" nature of a network environment, as put by Acemoglu et al. [2013] 11] Specifically, as shown in Theorem 3, when the underlying network structure passes a certain threshold, the trade pattern changes significantly. Adding more links to the network does not, however, further influence market behavior.

Below we provide the precise statement of the result in this section. The proof is given in Appendix A.2.

THEOREM 3 Given the network $G$ described as above, the following results hold:

- If $\frac{\left|S_{1}\right|}{|S|}<\frac{1+\frac{1}{\left|B_{1}\right|}}{2+\frac{1}{\left|B_{1}\right|}+\frac{1}{\left|B_{2}\right|}}$, then there exists $\delta_{1}<1$ such that when $\delta>\delta_{1}$, the market is segmented. At equilibrium, all sellers that are connected to both middlemen trade exclusively with 1, and their payoff decreases as $\left|S_{1}\right| /|S|$ increases.

- If $\frac{\left|S_{2}\right|}{|S|}<\frac{1+\frac{1}{\left|B_{2}\right|}}{2+\frac{1}{\left|B_{1}\right|}+\frac{1}{\left|B_{2}\right|}}$, then there exists $\delta_{2}<1$ such that when $\delta>\delta_{2}$, the market is segmented. At equilibrium all sellers that are connected to both middlemen trade exclusively with 2, and their payoff decreases as $\left|S_{2}\right| /|S|$ increases.

- Otherwise, if $\frac{\left|S_{1}\right|}{|S|} \geq \frac{1+\frac{1}{\left|B_{1}\right|}}{2+\frac{1}{\left|B_{1}\right|}+\frac{1}{\left|B_{2}\right|}}$ and $\frac{\left|S_{2}\right|}{|S|} \geq \frac{1+\frac{1}{\left|B_{2}\right|}}{2+\frac{1}{\left|B_{1}\right|}+\frac{1}{\left|B_{2}\right|}}$, then when $\delta>\max \left\{\delta_{1}, \delta_{2}\right\}$, the market is not segmented, and sellers will trade with both middlemen if they are connected. When $\delta$ approaches 1 , the agents' payoffs do not depend on $\left|S_{1}\right|,\left|S_{2}\right|$. Specifically, all the sellers have the same payoff of

$$
V_{s}=\frac{\left(\left|B_{1}\right|+1\right)\left(\left|B_{2}\right|+1\right)+\left|B_{1}\right|\left|B_{2}\right|-1}{(|S|+1)\left(\left|B_{1}\right|+1\right)\left(\left|B_{2}\right|+1\right)+\left|B_{1}\right|\left|B_{2}\right|-1} .
$$

The payoffs of buyers connected to middlemen 1 and 2 are

$$
V_{b_{1}}=\frac{|S|\left(\left|B_{2}\right|+1\right)}{(|S|+1)\left(\left|B_{1}\right|+1\right)\left(\left|B_{2}\right|+1\right)+\left|B_{1}\right|\left|B_{2}\right|-1} \text { and } V_{b_{2}}=\frac{|S|\left(\left|B_{1}\right|+1\right)}{(|S|+1)\left(\left|B_{1}\right|+1\right)\left(\left|B_{2}\right|+1\right)+\left|B_{1}\right|\left|B_{2}\right|-1}
$$

\footnotetext{
${ }^{11}$ Similar phenomena have been observed in other settings, such as financial contagion networks as in Acemoglu et al. [2013] and process flexibility as in Chou et al. [2010].
} 


$$
V_{m_{1}}=\frac{|S|\left|B_{1}\right|\left(\left|B_{2}\right|+1\right)}{(|S|+1)\left(\left|B_{1}\right|+1\right)\left(\left|B_{2}\right|+1\right)+\left|B_{1}\right|\left|B_{2}\right|-1} \text { and } V_{m_{2}}=\frac{|S|\left|B_{2}\right|\left(\left|B_{1}\right|+1\right)}{(|S|+1)\left(\left|B_{1}\right|+1\right)\left(\left|B_{2}\right|+1\right)+\left|B_{1}\right|\left|B_{2}\right|-1}
$$

\subsection{General Trading Networks and Traffic Routing Games}

Next, we consider a general trading network involving middlemen. In this setting, pairs of sellers and buyers trade in a network via one or multiple middlemen. In particular, we define the feasible coalitions within a network of sellers, buyers and middlemen as the groups of agents that form a path connecting a seller and a buyer. Using the convex program characterization, we show that the payoffs of the bargaining game coincides with the concept of congestion in an associated traffic routing game. The equivalence between the two seemingly unrelated games provides new insights into the influence of an agent's position in the network on her bargaining power 12

In particular, from the seminal work of Rubinstein [1982], we know that agents who make offers more frequently receive a higher share of the surplus. Thus, the rate at which agents make offers can be interpreted as their bargaining power. This intuition no longer holds in a networked market. Because of heterogeneous trade opportunities, an agent's bargaining power depends on the neighbors with whom she can trade. The neighbors' bargaining power also depends on their neighbors. This gives rise to a network effect in which an agent's ultimate payoff depends on the global structure of the network. One important consequence of this effect, as shown in Corominas-Bosch [2004] and Manea [2011], is that some trade opportunities are redundant. This is because, intuitively, two agents in strong positions can do better by waiting for opportunities to bargain with different agents in weaker position; thus they will never reach an agreement 13 Manea [2011], for example, takes advantage of this property to eliminate such redundant links and decompose the market into sub-markets.

\footnotetext{
${ }^{12} \mathrm{~A}$ number of researchers, such as Holme 2003] and Singh and Gupte [2005], have shown that betweenness centrality, a frequently used measure of centrality within a network, exhibits a strong correlation with the congestion level.

${ }^{13}$ Such a property is in sharp contrast to Ballester et al. [2006] and Golub and Elliott [2013], where an agent with strong neighbors obtains a high payoff because of complementaries.
} 
He shows that in each of the sub-markets, an agent's payoff depends on a statistic called shortage ratio.

As shown in the previous section, in the presence of middlemen, the market outcome is more complex. How exactly does an agent's share of surplus depend on the network structure? Our answer lies in the mapping between the bargaining game and a traffic routing game, where each coalition of the bargaining game corresponds to a route between an origin and a destination. Using the dual of the convex program, we show that agents' bargaining power coincides with the traffic flow through them in the traffic routing game. Intuitively, at equilibrium in a traffic routing game, only the fastest routes between an origin and a destination are taken. Also, from the convex program characterization, all the slower routes correspond to redundant coalitions that never reach agreement in the bargaining game. After eliminating these routes, agents with many routes flowing through them wait less time between making proposals to coalitions where agreement is profitable. These agents, therefore, are in relatively strong bargaining positions and are able to extract a higher share of surplus.

We now provide a formal description of our result in this section. We begin by assuming a network with the set of nodes $N$ and the set of links among some pairs of nodes $E \subset N \times N$. For ease of presentation, we assume the links are undirected; that is, if $(a, b) \in E$ then $(b, a) \in E$. Each link $e \in E$ is associated with a non-negative transaction cost $c_{e}$. The network is denoted by

$$
G=(N, E, \vec{c})
$$

We assume there are three types of nodes in this network: sellers, buyers and middlemen. There exists a set of seller-buyer pairs $\left\{\left(s_{k}, b_{k}\right): k \in K\right\}$, and each is associated with a non-negative trading surplus $V_{k} 14$ A path connecting a seller $s_{k}$ and a buyer $b_{k}$ is a sequence of nodes starting with $s_{k}$ and ending with $b_{k}$, such that every pair of consecutive nodes is connected by a link in the network. The cost of this path is the total transaction cost of all the links in the path.

\footnotetext{
${ }^{14}$ This general framework can model trade with heterogeneous goods, where a seller can only trade with some specific buyers. Homogeneous trade is a special case where the set $\left\{\left(s_{k}, b_{k}\right): k \in K\right\}$ contains all possible sellerbuyer pairs.
} 
The bargaining game is defined as in Section 2, where a feasible coalition is a set of agents who form a path connecting a seller $s_{k}$ and a buyer $b_{k}$, and the value of the coalition is $V_{k}$ minus the total cost of the path. Here we assume that agents have the same discount rate $\delta$. Furthermore, given a trading path $S$, all agents in $S$ have the same probability of being the proposer, i.e., a special case of the coalitional bargaining model where $\alpha_{i}=1$ for all $i$. This bargaining game is denoted by

$$
\mathcal{B}\left(G,\left\{\left(s_{k}, b_{k}, V_{k}\right): k \in K\right\}, P, \delta\right) .
$$

We now define a traffic routing game associated with the network bargaining game. Given the trading network $G=(N, E, \vec{c})$ and the seller-buyer pairs with trading surplus $\left\{\left(s_{k}, b_{k}, V_{k}\right): k \in K\right\}$, treat this network as a traffic network where each $\left(s_{k}, b_{k}\right)$ is an origindestination pair. The traffic in this network is modeled by the notion of flow, that is, a non-negative value on each path connecting an origin-destination pair. The flow of a path represents the amount of traffic traveling on it.

In particular, given a path $S$ let $f_{S}$ be its flow. Denote by $\vec{f}$ the vector of flows on all possible paths. For a given $\vec{f}$, let $x_{i}(\vec{f})$ be the total traffic going through node $i$; that is, $x_{i}(\vec{f})=\sum_{P: i \in P} f_{P}$.

The congestion at every link $e$ is given by the fixed $\operatorname{cost} c_{e}$. The congestion at node $i$ is equal to its total traffic $x_{i}(\vec{f})$. Given a path $S$ and a traffic flow $\vec{f}$, the congestion of the path $S$ is the total congestion of all nodes in $S$, and is denoted by $l(S, \vec{f})=\sum_{i: i \in S} x_{i}(\vec{f})+\sum_{e: e \in S} c_{e}$.

Given an origin-destination pair $\left(s_{k}, b_{k}\right)$, we assume that no single vehicle can influence the amount of traffic on any route, and all vehicles choose the route with the smallest total congestion. We further assume that for each origin-destination pair $\left(s_{k}, b_{k}\right), V_{k}$ is the maximum level of congestion that a vehicle going from $s_{k}$ to $b_{k}$ can tolerate, and there is always demand for traveling between $s_{k}$ and $b_{k}$ if there exists a connecting path of congestion less than $V_{k}$. In other words, the demand for traveling from $s_{k}$ to $b_{k}$ as a function of total congestion is inelastic with the cutoff of $V_{k}$. Hence, traffic flow $\vec{f}$ is in equilibrium if for any 
two paths $S, S^{\prime}$ connecting $s_{k}, b_{k}$, the following condition holds.

$$
f_{S}>0 \Longleftrightarrow V_{k}=l(S, \vec{f}) \leq l\left(S^{\prime}, \vec{f}\right)
$$

The traffic routing game described above is denoted by

$$
\mathcal{T}\left(G,\left\{\left(s_{k}, b_{k}, V_{k}\right): k \in K\right\}\right)
$$

The main result of this section is the following.

THEOREM 4 As $\delta$ approaches 1, the stationary equilibrium payoff of the bargaining game $\mathcal{B}\left(\delta, G,\left\{\left(s_{k}, b_{k}, V_{k}\right): k \in K\right\}\right)$ converges to the congestion level on the nodes of $G$ in the traffic routing game $\mathcal{T}\left(G,\left\{\left(s_{k}, b_{k}, V_{k}\right): k \in K\right\}\right)$.

Proof. See Appendix A.3.

We illustrate Theorem 4 by the following example.

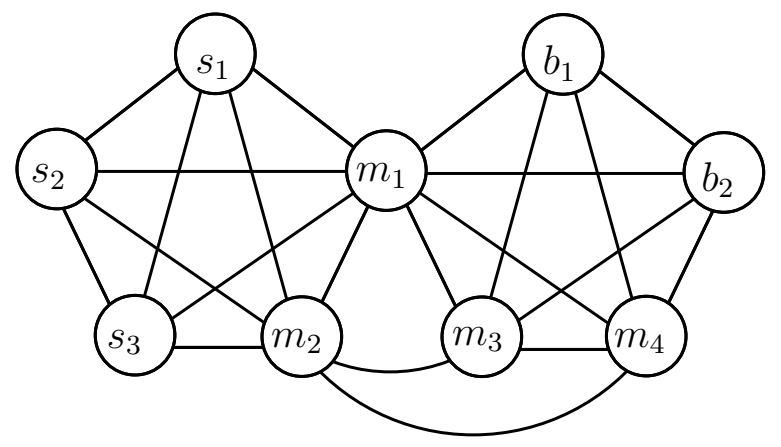

Figure 2: An example of trading network with middlemen

Consider the network of Figure 2, where the sellers are $s_{1}, s_{2}$ and $s_{3}$; the buyers are $b_{1}$ and $b_{2}$; and the middlemen are $m_{1}, m_{2}, m_{3}$ and $m_{4}$. Assume that the transaction costs for all links are 0, the set of seller-buyer pairs is all possible pairs of buyers and sellers, and the total trading surplus is 43 for all such pairs.

According to Theorem 2, as $\delta \rightarrow 1$, the outcome of this bargaining game converges to the unique solution of the following convex program

$$
\begin{aligned}
& \operatorname{minimize} \sum_{i \in N} u_{i}^{2} \\
& \text { subject to } \sum_{j \in S} u_{j} \geq 43 \quad \forall \text { seller-buyer paths } S .
\end{aligned}
$$


The unique solution of this convex program is given by the number in each node of the network in Figure 3. Here we obtain that $u_{m_{1}}=18 ; u_{m_{2}}=12 ; u_{m_{3}}=u_{m_{4}}=6$.

We now use the duality theorem of the convex program to further analyze this solution. Let $u$ be the optimal solution of (C), consider the dual $\lambda_{S}$ for each constraint $S$, which is a path that connects a seller and a buyer. By the Karush-Kuhn-Tucker condition we have

$$
\begin{aligned}
& u_{i}=\sum_{S: i \in S} \lambda_{S} \\
& \lambda_{S}\left(\sum_{i \in S} u_{i}-43\right)=0 .
\end{aligned}
$$

For each link $e$, consider the sum of the duals on all of the paths containing this link. Namely, $w_{e}=\sum_{S: e \in S} \lambda_{S} 15$ In Figure 3 , the weight $w_{e^{-S}}$ are given on the links of the network. For a dashed link $e, w_{e}=0$. Orient the links in the direction from sellers to buyers, as illustrated in Figure 3. Notice that for every middleman node, the total of the weights on the links coming into the node is equal to the total of the weights on the links going out.

Now, consider the following interpretation. Consider each node in the network as a city, each link as a highway, and $w_{e}$ as the amount of traffic on the the highway $e$. Because of (5) , for a city $i$, the total amount of traffic coming in (or going out) is equal to $u_{i}$. Interpret $u_{i}$ as the level of congestion at $i$. For a dashed link $e$, we have $w_{e}=0$, meaning there is no traffic on this link. This is because all seller-buyer paths going through this link have the total of $u_{i}$ (congestion) greater than 43 . Thus, the paths containing the dashed links can be interpreted as overly congested routes.

An insight we glean from this model is that not all links are essential in determining the outcome of the bargaining game 16 Notice that in Figure 3, on any seller-buyer path that contains a dashed link, the total of $u_{i}$ is greater than 43. In the bargaining game, these links will not be in any coalition that is formed at equilibrium. Deleting them from the network does not not influence the solution of the convex program, and thus will not influence the outcome of either the bargaining game or the traffic routing game.

\footnotetext{
${ }^{15}$ Notice that here a coalition $S$ corresponds to a path; thus, we also use the notation $e \in S$ if the link $e$ is contained in the path $S$.

${ }^{16}$ This property is consistent with Corominas-Bosch 2004] and Manea 2011.
} 


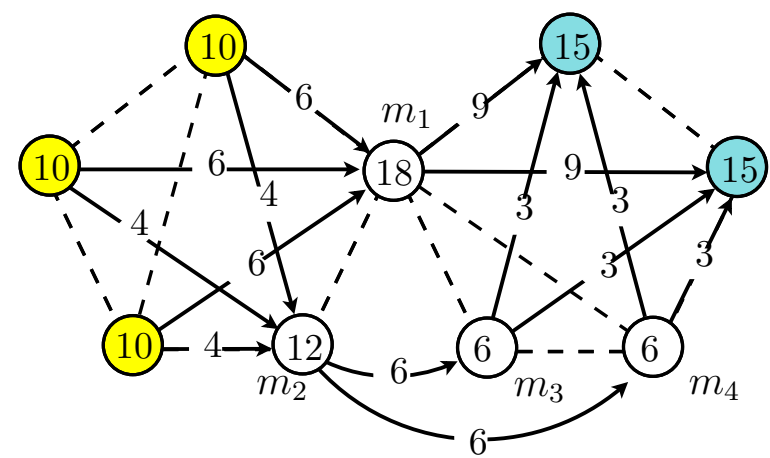

Figure 3: The bargaining solution.

The equivalence between the bargaining game and the traffic routing game, as shown in Figure 3, provides another qualitative insight. Namely, in that example, the size and the number of profitable coalitions that an agent belongs to are the two main factors determining her bargaining power. Intuitively, an agent in small coalitions gets a high payoff because she does not need to share surplus with many other agents. Moreover, an agent belonging to many profitable coalitions waits less time between making proposals, and thus has relatively strong bargaining power.

The combined effect of these two factors is perfectly captured in the congestion levels of the traffic routing game. In particular, consider the traffic network in Figure 3 after eliminating the dashed links. First, notice that all the short paths (with one hop) between a seller and a buyer go through $m_{1}$, while all the longer ones (with two hops) go through $m_{2}$. In the traffic routing game more vehicles prefer to travel through the shorter routes; thus, the congestion at $m_{1}$ is higher than at $m_{2}$. This translates to the fact that in the bargaining game, $m_{1}$ has a higher payoff, because unlike $m_{2}, m_{1}$ does not have to share the surplus with any middlemen. Second, observe that $m_{2}$ 's congestion is higher than both $m_{3}$ 's and $m_{4}$ 's. This is because the number of routes going through $m_{2}$ is equal to the number of routes going through $m_{3}$ and $m_{4}$ combined. Correspondingly, in the bargaining game, $m_{2}$ belongs to all the profitable coalitions that contains either $m_{3}$ or $m_{4}$. Therefore, $m_{2}$ is in a stronger bargaining position than both $m_{3}$ and $m_{4}$. 


\subsection{Cooperation in Overlapping Communities}

In our second application, we consider a setting where simultaneous trade among more than two agents is possible and modeled by a supermodular surplus function. Similar to the application in Section 4.2, the set of feasible coalitions is also determined by an underlying network.

Let $G$ be a network with the set of nodes $N$ and the set of links $E \subset N \times N$. The nodes of $G$ represent the agents, and the links correspond to a mutual relationship between the agents.

A feasible coalition is a set of nodes $S \subset N$ such that there is a link between every pair of nodes in $S$. As an example of this model, when the links of $G$ represent either a social connection or geographical closeness between the two agents, a feasible coalition corresponds to a group of people that belong to the same social or geographical community.

The characteristic function $V: 2^{N} \rightarrow \mathbb{R}$ is assumed to be strictly supermodular, that is $V(A)+V(B)<V(A \cup B)+V(A \cap B)$, for all $A, B \subset N$ such that both $A \backslash B$ and $B \backslash A$ are nonempty. Define the game $\Gamma(G, V, \delta)$ as the bargaining game as in Section 2, where the feasible coalitions are sets of pairwise connected nodes in the network $G 17$

A set of pairwise connected nodes is called a clique in network theory. Notice that for a clique $S$, any $S^{\prime} \subset S$ is also a clique. A clique $S$ is a maximal clique if there is no larger clique that contains $S$. As an example, the network in Figure 2 contains 3 maximal cliques: $\left\{s_{1}, s_{2}, s_{3}, m_{1}, m_{2}\right\},\left\{b_{1}, b_{2}, m_{1}, m_{3}, m_{4}\right\}$ and $\left\{m_{1}, m_{2}, m_{3}, m_{4}\right\}$. We call each maximal clique a community. In the example in Figure2, there are three communities, and agents can belong to multiple communities. In other words, these communities can overlap.

Our main finding is that hierarchical structures emerge endogenously as an outcome of the bargaining game. In particular, we show that the set of coalitions that are formed at equilibrium in a given community has a "nesting" structure, that is one coalition contains another. See Figure 4 for an illustration. In that figure, there are three communities, represented by three largest circles, the circles represent the set of coalitions formed at equilibrium. In each

\footnotetext{
${ }^{17}$ Kets et al. 2011] study a similar environment in a cooperative game theory framework.
} 
community, the coalitions formed at equilibrium contain one another. One can interpret the nesting property of the formed coalition in each community as a hierarchy; agents that belong to a smaller coalition in some sense are more "important" and have higher bargaining power. In particular, our result shows that an agent's bargaining power (the outcome of the corresponding bargaining game) is a weighted sum over the set of circles to which she belongs. This result demonstrates that in this setting, an agent's bargaining power depends on the combination of two factors: the position of the agent in the hierarchy and the number of communities the agent belongs to.

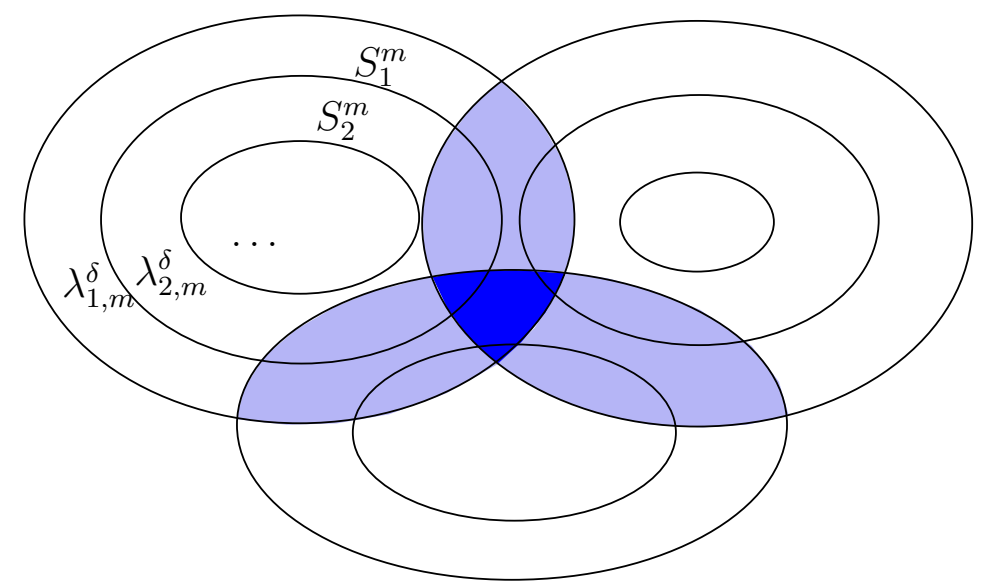

Figure 4: Hierarchal structure of the forming coalition in each community

Our main results in this section are given by Proposition 4.1 and Corollary 4.2 ,

PROPOSITION 4.1 Consider $\Gamma(G, V, \delta)$, where $V$ is strictly supermodular. Let $u_{i}^{* \delta}$ be the payoff of agent $i$ and let $C_{1}, \ldots, C_{M}$ be the set of communities (maximal clique) of $G$. Then there exists $0<\delta_{0}<1$, and for each community $C_{m}$ a set of nesting coalitions $C_{m} \supset S_{1}^{m} \supsetneq$ $S_{2}^{m} \supsetneq \cdots \supsetneq S_{K_{m}}^{m}$, such that the following holds. For all $\delta>\delta_{0}$ there exist nonnegative $\lambda_{k, m}^{\delta}$ associated with the coalition $S_{k}^{m}$ where $u_{i}^{* \delta}=\sum_{m, k: i \in S_{k}^{m}} \lambda_{k, m}^{\delta}$. Furthermore, all coalitions other than these $S_{k}^{m}$ will not be formed in the bargaining game $\Gamma(G, V, \delta)$ for $\delta>\delta_{0}$.

Proof. See Appendix A.4.

To illustrate, consider the case when the characteristic function $V(S)$ on each maximal 
clique only depends on the size of the coalition. The following corollary shows that agents belonging to more communities will have higher payoffs.

COROLLARY 4.2 Consider the game $\Gamma(G, V, \delta)$, assume $V(S)$ is a function of $|S|$. Consider two agents $i$ and $j$ such that $i$ belongs to all communities that $j$ is a member of, then $i$ 's payoff is at least as $j$ 's, that is $u_{i}^{* \delta} \geq u_{j}^{* \delta}$.

Proof. See Appendix A.5.

\section{Conclusion}

We introduce a model of non-cooperative bargaining in general coalition formation games, and provide a new method using convex programming to characterize the stationary equilibrium. We apply this framework to several network games. In a trading network involving middlemen we prove the equivalence between the outcomes of the bargaining game and the corresponding traffic network. In the application of cooperation networks, we show the endogenous emergence of hierarchical structures among the agents. These results provide new insights into the influence of an agent's position in the network on her bargaining power. Importantly, the new approach to characterize the unique equilibrium payoff that we introduce in our paper can likely be applied to other economic and market settings that have previously been difficult to model.

\section{Acknowledgments}

The author thanks Rakesh Vohra, Randall Berry, Mihai Manea, two anonymous referees, and the associate editor for their insightful comments and suggestions. I am especially grateful to one of the referees for suggesting an insightful and elegant way of interpreting the connection with traffic routing games. 


\section{References}

D. Abreu and M. Manea. Bargaining and efficiency in networks. J. Economic Theory, 147 (1):43-70, 2012a.

D. Abreu and M. Manea. Markov equilibria in a model of bargaining in networks. Games and Economic Behavior, 75(1):1-16, 2012b.

D. Acemoglu, A. Ozdaglar, and A. Tahbaz-Salehi. Systemic risk and stability in financial networks. Working Paper, 2013.

V. Bala and S. Goyal. A noncooperative model of network formation. Econometrica, 68(5): 1181-1229, 2002.

C. Ballester, A. Calv-Armengol, and Y. Zenou. Who's who in networks. wanted: The key player. Econometrica, 74(5):1403-1417, 092006.

R. S. Burt. Structural holes : the social structure of competition. Harvard University Press, 1992.

M. C. Chou, G. A. Chua, C.-P. Teo, and H. Zheng. Design for process flexibility: Efficiency of the long chain and sparse structure. Operations Research, 58(1):43-58, 2010.

D. Condorelli and A. Galeotti. Bilateral trading in networks. Working paper, 2014.

M. Corominas-Bosch. Bargaining in a network of buyers and sellers. J. Economic Theory, 115(1):35-77, 2004.

P. Csóka, P. J.-J. Herings, and L. A. Kóczy. Stable allocations of risk. Games and Economic Behavior, 67(1):266-276, 2009.

M. Elliott. Inefficiencies in networked markets. American Economic Journal: Microeconomics, 2014. 
D. Gale. Limit theorems for markets with sequential bargaining. Journal of Economic Theory, 43(1):20-54, October 1987.

M. Gofman. A network-based analysis of over-the-counter markets. Working paper, 2011.

B. Golub and M. Elliott. A network approach to public goods. Working paper, 2013.

S. Goyal and F. Vega-Redondo. Structural holes in social networks. Journal of Economic Theory, 137(1):460-492, November 2007.

P. Holme. Congestion and centrality in traffic flow on complex networks. Advances in Complex Systems, 6(2):163-176, 2003.

M. O. Jackson. Social and Economic Networks. Princeton University Press, Princeton, NJ, USA, 2008.

M. O. Jackson and A. Wolinsky. A strategic model of social and economic networks. Journal of Economic Theory, 71(1):44-74, October 1996.

L. V. Kantorovich. Mathematical methods of organizing and planning production. Management Science, 6(4):pp. 366-422, 1960.

W. Kets, G. Iyengar, R. Sethi, and S. Bowles. Inequality and network structure. Games and Economic Behavior, In Press, Corrected Proof:-, 2011.

T. C. Koopmans. Optimum utilization of the transportation system. Econometrica, 17:pp. 136-146, 1949.

R. E. Kranton and D. F. Minehart. A theory of buyer-seller networks. American Economic Review, 91(3):485-508, June 2001.

L. Lovász and M. Plummer. Matching Theory. Akadémiai Kiadó-Budapest, 1986.

M. Manea. Bargaining in stationary networks. American Economic Review, 101(5):2042-80, August 2011. 
M. Manea. Bargaining in dynamic markets. Working paper, 2013a.

M. Manea. Steady states in matching and bargaining. Working paper, 2013b.

M. Manea. Intermediation in networks. working paper, 2013c.

R. B. Myerson. Graphs and cooperation in games. Mathematics of Operations Research, 2 (3):pp. 225-229, 1977.

T. Nguyen. Local bargaining and supply chain instability. Working paper, October 2013.

R. T. Rockafellar. Convex Analysis. Princeton University Press, Princeton, NJ, 1970.

A. Rubinstein. Perfect equilibrium in a bargaining model. Econometrica, 50(1):pp. 97-109, 1982.

A. Rubinstein and A. Wolinsky. Equilibrium in a market with sequential bargaining. Econometrica, 53(5):1133-50, September 1985.

A. Rubinstein and A. Wolinsky. Middlemen. The Quarterly Journal of Economics, 102(3): 581-93, August 1987.

A. Schrijver. Handbook of Discrete Optimization, chapter On the history of combinatorial optimization (till 1960), pages 1-68. editorsK. Aardal, G.L. Nemhauser, R., Elsevier, Amsterdam, 2005.

J.-P. Siedlarek. Intermediation in networks. Working Paper, 2012.

B. K. Singh and N. Gupte. Congestion and decongestion in a communication network. Phys. Rev. E, 71:055103, May 2005.

Y.-Y. Wong and R. Wright. Buyers, sellers and middlemen:variations on search-theoretic themes. International Economic Review, 55:375-398, 2014. 


\section{A Missing Proofs}

\section{A.1 Proof of Theorem 2}

In this proof, we denote the sum $\sum_{i} \frac{1}{\alpha_{i}} u_{i}^{2}$ by $\|u\|_{\alpha}^{2}$. Recall that $u^{*}$ is the optimal solution of (B) and $u^{* \delta}, z_{S}^{\delta}$ is the optimal solution of ( $\left(\mathrm{A}^{\prime}\right)$.

Consider $u_{i}=\frac{1}{\delta} u_{i}^{*}, z_{S}=0$, it is straightforward to see that $(\vec{u}, \vec{z})$ is a feasible solution of (A'), for which $u^{* \delta}, z_{S}^{\delta}$ is the optimal solution. Therefore,

$$
\left\|\frac{u_{i}^{*}}{\delta}\right\|_{\alpha}^{2}=\sum \frac{1}{\alpha_{i}}\left(\frac{u_{i}^{*}}{\delta}\right)^{2}+0 \geq \sum_{i \in N} \frac{1}{\alpha_{i}}\left(u_{i}^{* \delta}\right)^{2}+\frac{1}{\delta(1-\delta)} \sum_{S \in \mathcal{F}} P(S) \cdot\left(z_{S}^{\delta}\right)^{2} \geq\left\|u^{* \delta}\right\|_{\alpha}^{2} .
$$

This implies

$$
\frac{1}{\delta}\left\|u^{*}\right\|_{\alpha} \geq\left\|u^{* \delta}\right\|_{\alpha}
$$

When $\delta$ tends to $1, z_{S}^{\delta}$ approaches 0 because otherwise, the objective of (A') approaches $\infty$, which contradicts the fact that it is the optimal solution of (A'). Therefore, there exists $\epsilon(\delta)$ such that $\lim _{\delta \rightarrow 1} \epsilon(\delta)=0$ and $z_{S}^{\delta}<\epsilon(\delta)$. Because of the constraints in (A'), we have

$$
\sum_{i \in S} u_{i}^{* \delta} \geq V(S)-\epsilon(\delta) \forall S \in \mathcal{F}
$$

Note that $u^{*}$ is the unique optimal solution of $(\underline{B})$; thus, $u^{*}$ is the unique point in the intersection of two sets

$$
\left\{\vec{u}:\|u\|_{\alpha} \leq\left\|u^{*}\right\|_{\alpha}\right\} \text { and }\left\{\vec{u}: \sum_{i \in S} u_{i} \geq V(S) \forall S\right\}
$$

As $\delta$ approaches $1,\left\{\vec{u}:\|u\|_{\alpha} \leq \frac{1}{\delta}\left\|u^{*}\right\|_{\alpha}\right\}$ converges to $\left\{\vec{u}:\|u\|_{\alpha} \leq\left\|u^{*}\right\|_{\alpha}\right\}$ and $\left\{\sum_{i \in S} u_{i} \geq\right.$ $V(S)-\epsilon(\delta) \quad \forall S\}$ converges to $\left\{\vec{u}: \sum_{i \in S} u_{i} \geq V(S) \quad \forall S\right\} 18$ Thus, the intersection of $\left\{\vec{u}:\|u\|_{\alpha} \leq \frac{1}{\delta}\left\|u^{*}\right\|_{\alpha}\right\}$ and $\left\{\vec{u}: \sum_{i \in S} u_{i} \geq V(S)-\epsilon(\delta) \quad \forall S\right\}$ shrinks to the single point $u^{*}$. Because of (7) and (8),$u^{* \delta}$ is in this intersection. This shows that $u^{* \delta} \stackrel{\delta \rightarrow 1}{\longrightarrow} u^{*}$. The second part of the theorem follows because $\lim _{\delta \rightarrow 1} u^{* \delta}=u^{*}$ and $\lim _{\delta \rightarrow 1} z^{\delta}=0$.

\footnotetext{
${ }^{18}$ Here, we consider the Hausdorff distance between the two sets.
} 


\section{A.2 Proof of Theorem 3}

Consider a seller who is connected to both of the middlemen, as $\delta$ approaches 1 , there are three possible patterns of trade that can emerge as the outcome of the game. First, the seller only trades with middleman 1 ; second, the seller only trades with middleman 2; lastly, the seller trades with both middlemen. Notice that at equilibrium, the payoffs to the seller in each of the three scenarios are strictly positive, and thus the seller will trade with at least one of the middlemen. For each of the first two cases, we derive the conditions on $|S|,\left|S_{1}\right|,\left|S_{2}\right|$, $\left|B_{1}\right|$ and $\left|B_{2}\right|$ for the corresponding trade pattern to be an equilibrium outcome. When the parameters do not satisfy the conditions necessary for the first two equilibrium outcomes, it must be true that the third outcome emerges at equilibrium, and the seller will trade with both middlemen. After we determine the outcome of the game, we compute the equilibrium payoffs to the agents.

We first need the following lemma.

LEMMA A.1 Consider a network with s sellers and b buyers connected through a single middleman. As $\delta \rightarrow 1$, the equilibrium payoffs approach $V_{s}=\frac{b}{b+s+b s}$ for the sellers; $V_{b}=$ $\frac{s}{b+s+b s}$ for the buyers; and $V_{m}=\frac{b s}{b+s+b s}$ for the middleman.

Proof. Proof of Lemma A.1 Because of symmetry, let $V_{s}, V_{b}$ and $V_{m}$ be the payoff of a seller, a buyer and the middleman. According to Theorem 2, the limit equilibrium payoff is the solution of the following optimization problem

$$
\begin{gathered}
\min s \cdot V_{s}^{2}+b \cdot V_{b}^{2}+V_{m}^{2} \\
\text { s.t } V_{s}+V_{b}+V_{m} \geq 1
\end{gathered}
$$

The solution of this convex program is exactly the value given in Lemma A.1.

Consider the first case: no seller connected to both middlemen trades with 2 . In such a case the market is segmented into 2 submarkets. In the first one, $\left|S_{1}\right|$ sellers and $\left|B_{1}\right|$ buyers are connected to the middleman 1. In the second market $|S|-\left|S_{1}\right|$ sellers and $\left|B_{2}\right|$ buyers are connected to the middleman 2. According to Lemma A.1, Let $V_{s_{i}}, V_{m_{i}}$, and $V_{b_{i}}$ be the payoff 
of a seller, a middlemen, and a buyer in the sub-market $i \in\{1,2\}$. Notice that all sellers connected to both middlemen choose to trade with middleman 1 only. From Theorem 2 , this holds if and only if $V_{s_{1}}+V_{m_{2}}+V_{s_{2}}>1$. This equivalent to $V_{s_{1}}=\frac{\left|B_{1}\right|}{\left|S_{1}\right|+\left|B_{1}\right|+\left|S_{1}\right|\left|B_{1}\right|}>$ $\frac{\left|B_{2}\right|}{|S|-\left|S_{1}\right|+\left|B_{2}\right|+\left(|S|-\left|S_{1}\right|\right)\left|B_{2}\right|}=V_{s_{2}}$. From this we get

$$
\frac{\left|S_{1}\right|}{|S|}<\frac{1+\frac{1}{\left|B_{1}\right|}}{2+\frac{1}{\left|B_{1}\right|}+\frac{1}{\left|B_{2}\right|}} .
$$

Similarly, for case 2, no seller connected to both middlemen trades with 1 , and thus, we get $\frac{\left|B_{2}\right|}{\left|S_{2}\right|+\left|B_{2}\right|+\left|S_{2}\right|\left|B_{2}\right|}>\frac{\left|B_{1}\right|}{|S|-\left|S_{1}\right|+\left|B_{1}\right|+\left(|S|-\left|S_{1}\right|\right)\left|B_{1}\right|}$. This is equivalent to

$$
\frac{\left|S_{2}\right|}{|S|}<\frac{1+\frac{1}{\left|B_{2}\right|}}{2+\frac{1}{\left|B_{1}\right|}+\frac{1}{\left|B_{2}\right|}} .
$$

When (9) and (10) do not hold, then sellers connected to both middlemen will trade with both of them. In this case, we will show that all sellers obtain the same payoff in the limit as $\delta \rightarrow 1$. Let $V_{s_{1}}$ and $V_{s_{2}}$ be the payoff of the sellers that are only connected to either middleman 1 or 2 , respectively, and let $V_{s_{12}}$ be the payoff of the sellers that are connected to both. For buyers and middlemen, we use the same notation as above. Now, because sellers that are connected to both middlemen trade with both of them, due to Theorem 2, we have $V_{s_{12}}+V_{m_{1}}+V_{b_{1}}=1$. However, we also have $V_{s_{1}}+V_{m_{1}}+V_{b_{1}}=1$. Thus, $V_{s_{12}}=V_{s_{1}}$. Similarly, $V_{s_{12}}=V_{s_{2}}$. From this we obtain $V_{s_{12}}=V_{s_{1}}=V_{s_{2}}=V_{s}$.

Therefore, in this case, the equilibrium payoff at the limit as $\delta \rightarrow 1$ is the solution of the following program:

$$
\begin{aligned}
& \min |S| \cdot V_{s}^{2}+\left|B_{1}\right| \cdot V_{b_{1}}^{2}+\left|B_{2}\right| \cdot V_{b_{2}}^{2}+V_{m_{1}}^{2}+V_{m_{2}}^{2} \\
& \text { s.t } V_{s}+V_{b_{i}}+V_{m_{i}} \geq 1 \text { for } i=1,2 .
\end{aligned}
$$

Solving this program, we obtain

$$
\begin{gathered}
V_{s}=\frac{\left(\left|B_{1}\right|+1\right)\left(\left|B_{2}\right|+1\right)+\left|B_{1}\right|\left|B_{2}\right|-1}{(|S|+1)\left(\left|B_{1}\right|+1\right)\left(\left|B_{2}\right|+1\right)+\left|B_{1}\right|\left|B_{2}\right|-1} ; \\
V_{b_{1}}=\frac{|S|\left(\left|B_{2}\right|+1\right)}{(|S|+1)\left(\left|B_{1}\right|+1\right)\left(\left|B_{2}\right|+1\right)+\left|B_{1}\right|\left|B_{2}\right|-1} ; V_{b_{2}}=\frac{|S|\left(\left|B_{1}\right|+1\right)}{(|S|+1)\left(\left|B_{1}\right|+1\right)\left(\left|B_{2}\right|+1\right)+\left|B_{1}\right|\left|B_{2}\right|-1} ; \\
V_{m_{1}}=\frac{|S|\left|B_{1}\right|\left(\left|B_{2}\right|+1\right)}{(|S|+1)\left(\left|B_{1}\right|+1\right)\left(\left|B_{2}\right|+1\right)+\left|B_{1}\right|\left|B_{2}\right|-1} ; V_{m_{2}}=\frac{|S|\left|B_{2}\right|\left(\left|B_{1}\right|+1\right)}{(|S|+1)\left(\left|B_{1}\right|+1\right)\left(\left|B_{2}\right|+1\right)+\left|B_{1}\right|\left|B_{2}\right|-1} .
\end{gathered}
$$




\section{A.3 Proof of Theorem 4}

According to Theorem 2, when $\delta \rightarrow 1$, the stationary equilibrium payoff of the bargaining game $\mathcal{B}\left(\delta, G,\left\{\left(s_{k}, b_{k}, V_{k}\right): k \in K\right\}\right)$ converges to the optimal solution of the following program

$$
\begin{aligned}
\text { minimize: } & \sum_{i \in N} u_{i}^{2} \\
\text { subject to: } & \sum_{i \in S} u_{i} \geq V_{k}-\sum_{e \in S} c_{e} \text { for all path } S \text { connecting } s_{k}, b_{k} .
\end{aligned}
$$

Due to Lemma 3.1, $u^{*}$ is the optimal solution of (11) if and only if there exist $\lambda_{S} \geq 0$ such that $u_{i}^{*}=\sum_{S: i \in S} \lambda_{S}$. Furthermore, for a path $S$ connecting $s_{k}, b_{k}$

$$
\text { if } \sum_{i \in S} u_{i}^{*}>V_{k}-\sum_{e \in S} c_{e} \text {, then } \lambda_{S}=0 \text { and if } \lambda_{S}>0 \text {, then } \sum_{i \in S} u_{i}^{*}=V_{k}-\sum_{e \in S} c_{e} \text {. }
$$

This condition is exactly the condition for $\vec{\lambda}$ to be an equilibrium flow of $\mathcal{T}\left(G,\left\{\left(s_{k}, b_{k}, V_{k}\right)\right.\right.$ : $k \in K\})$. Thus, the congestion for $i$ coincides with $u_{i}^{*}$.

\section{A.4 Proof of Proposition 4.1}

Let $u^{*}$ be the limit payoff of $u^{* \delta}$, then $u^{*}$ is the optimal solution of

$$
\begin{gathered}
\min \sum_{i \in N} u_{i}^{2} \\
\text { subject to } \sum_{j \in S} u_{j} \geq V(S) \quad \forall S \in \mathcal{F}
\end{gathered}
$$

The proof is based on Theorem 2 and Lemma 3.2. We know that the program (B) characterizes the payoff at the limit $\delta \rightarrow 1$.

Given a community $C_{m}$, let $S_{1}^{m}, S_{2}^{m}, \ldots, S_{K}^{m}$ be the subsets of $C_{m}$ of which the corresponding constraints bind in the program $(\underline{B})$.

We first show that these sets are nested. Assume the contrary, let $S, T$ be the two such binding sets which are not nested, that is the two sets $S \cap T, S \cup T$ are different from $S, T$.

Let $u^{*}$ be the solution of the optimization program above, then

$$
V(S)+V(T)=\sum_{i \in S} u_{i}^{*}+\sum_{i \in T} u_{i}^{*}=\sum_{i \in S \cap T} u_{i}^{*}+\sum_{i \in S \cup T} u_{i}^{*}
$$


Because both $S, T$ are subset of $C_{m}$, both $S \cap T, S \cup T$ are also subsets of $C_{m}$, which means they are both cliques. Thus, according to the constraints in the optimization program we have

$$
\sum_{i \in S \cap T} u_{i}^{*}+\sum_{i \in S \cup T} u_{i}^{*} \geq V(S \cap T)+V(S \cup T) .
$$

These inequalities imply $V(S)+V(T) \geq V(S \cap T)+V(S \cup T)$, which contradicts to the strict supermodularity of $V$.

Now, $u^{* \delta}$ is the stationary equilibrium payoff of $(G, V, \delta)$. Thus, it is the optimal solution of A'] According to Lemma 3.2, there exists $\lambda_{S}^{\delta}$ such that $u_{i}^{* \delta}=\sum_{k: i \in S} \lambda_{S}^{\delta}$, and $\lambda_{S}^{\delta}=0$ if $S$ is not formed in the game $(N, V, \delta)$.

Using Theorem 2, there exists $\delta_{0}$ such that if $\delta>\delta_{0}$, then a set that is not one of $S_{k}$ will not be formed at an equilibrium of $(G, V, \delta)$. Thus, $\lambda_{S}^{\delta}=0$ if $S \notin\left\{S_{1}, \ldots, S_{K}\right\}$. This concludes the proof.

\section{A.5 Proof of Corollary 4.2}

Assume $u_{i}^{* \delta}<u_{j}^{* \delta}$, then because of Lemma 3.2, there is a binding coalition $S$ that contains $j$ but not $i$. Because $i$ is in all the communities that $j$ belongs to, thus $S^{\prime}=S-\{j\} \cup\{i\}$ is also a feasible coalition. However,

$$
\sum_{k \in S^{\prime}} u_{k}^{* \delta}<\sum_{k \in S} u_{k}^{* \delta}=V(S)=V\left(S^{\prime}\right)
$$

The last inequality holds because $v$ only depends on the size of the coalition. But this is a contradiction to $\sum_{k \in S^{\prime}} u_{k}^{* \delta} \geq V\left(S^{\prime}\right)$, which is the constraint in the program (A').

\section{B Formation of Seller-Buyer Networks}

Consider a seller-buyer network with a set of sellers and buyers denoted by $S$ and $B$, respectively. Let $\mathcal{F} \subset S \times B$ be the set of feasible trade between sellers and buyers. We assume each seller has an indivisible good to sell, and buyers have unit demand. Let $V_{i j}>0$, for $(i, j) \in \mathcal{F}$ be the total surplus if a buyer $j$ obtains the good from a seller $i$. Unlike the model 
of Manea, here, we assume $V_{i j}$ are different for different $i, j$. Furthermore, we assume these surpluses are generic, which is defined as follows.

DEFINITION 1 A vector $\vec{V} \in \mathbb{R}^{m}$ is generic if there do not exist two disjoint subsets of indexes $M_{1}, M_{2} \subset[1, \ldots, m]$ such that $\sum_{k \in M_{1}} V_{k}=\sum_{l \in M_{2}} V_{l}$.

Consider the coalitional bargaining game with the set of feasible coalitions $\mathcal{F}$ and the surplus function $V(i, j)=V_{i j}$, described by the seller-buyer network as above. We say a link $(i, j)$ is formed if at the equilibrium of the bargaining game if the pair $i, j$ trade with a positive probability. Our result is the following.

THEOREM 5 Given a seller-buyer network bargaining game described above, there exist $0<\delta_{1}<\delta_{2}<1$ such that, when $\delta<\delta_{1}$ all pairs $(i, j) \in \mathcal{F}$ are formed at equilibrium. However, when $\delta>\delta_{2}$ the subnetwork formed at equilibrium is a forest 19

Proof. Proof: According to Theorem 1, the payoff $\vec{u}$ of the bargaining game is characterized by the following convex program 20

$$
\begin{aligned}
\text { minimize: } & \sum_{i \in N} \delta(1-\delta) u_{i}^{2}+\sum_{(i, j) \in \mathcal{F}} P(i, j) z_{i j}^{2} \\
\text { subject to: } & \delta u_{i}+\delta u_{j}+z_{i j} \geq V_{i j} \quad \forall(i, j) \in \mathcal{F}, \\
& u_{i} \geq 0, z_{i j} \geq 0 \quad \forall i \in N,(i, j) \in \mathcal{F} .
\end{aligned}
$$

We will show that as $\delta$ approaches 0 , all variables $z_{i j}$ are positive in the optimal solution of (12). Due to Theorem 1, all links $i j \in \mathcal{F}$ are formed at equilibrium.

To see this, we introduce $w_{i}=\delta u_{i}$. Thus, (12) becomes

$$
\begin{aligned}
\operatorname{minimize}: & \sum_{i \in N} \frac{(1-\delta)}{\delta} w_{i}^{2}+\sum_{(i, j) \in \mathcal{F}} P(i, j) z_{i j}^{2} \\
\text { subject to: } & w_{i}+w_{j}+z_{i j} \geq V_{i j} \quad \forall(i, j) \in \mathcal{F}, \\
& w_{i} \geq 0, z_{i j} \geq 0 \quad \forall i \in N,(i, j) \in \mathcal{F} .
\end{aligned}
$$

\footnotetext{
${ }^{19} \mathrm{~A}$ forest is a sparse undirected graph, which consists of disjoint trees. An equivalent condition is that it does not contain any cycle.

${ }^{20}$ Recall we assume $\alpha_{i}=1$, and $\delta_{i}=\delta$ for all $i$.
} 
Let $w^{*}, z^{*}$ be the optimal solution of (13). As $\delta$ approaches 0 , the coefficient of $w_{i}, \frac{1-\delta}{\delta}$, approaches infinity. However, the optimal solution of (13) is bounded from above 21 Therefore, $w_{i}^{*}$ approaches 0 as $\delta$ tends to 0 for all $i$. Thus, there exists $\delta_{1}>0$ such that when $\delta<\delta_{1}$, $w_{k}^{*}<\min _{i j}\left\{V_{i j} / 2\right\}$ for all $k$. This implies $z_{i j}^{*} \geq V_{i j}-w_{i}^{*}-w_{j}^{*}>0$. By this we prove that all links are formed with probability 1 when $\delta<\delta_{1}$.

To prove the second half of the theorem, consider the following limit program

$$
\begin{aligned}
\operatorname{minimize}: & \sum_{i \in N} u_{i}^{2} \\
\text { subject to: } & u_{i}+u_{j} \geq V_{i j} \quad \forall(i, j) \in \mathcal{F}, \\
& u_{i} \geq 0 \quad \forall i \in N,(i, j) \in \mathcal{F} .
\end{aligned}
$$

Let $u^{*}$ be the optimal solution of this program. Due to Theorem 2, there exists $\delta_{2}<1$ such that when $\delta>\delta_{2}$, all links in which $u_{i}^{*}+u_{j}^{*}>V_{i j}$ are not formed at equilibrium. Thus, we will consider the subnetwork consisting of links $i, j$ for which $u_{i}^{*}+u_{j}^{*}=V_{i j}$. We will show that there cannot be any cycle in this network, and thus it is a forest.

Assume that there exists a cycle $1,2, \ldots, k$ that is formed at the equilibrium. Because the underlying seller-buyer network is bipartite, the length of the cycle $k$ is even.

We have $u_{i}^{*}+u_{j}^{*}=V_{i j}$ for all links in the cycle, therefore, $\sum_{i=1}^{k} u_{i}^{*}=\left(u_{1}^{*}+u_{2}^{*}\right)+\left(u_{3}^{*}+u_{4}^{*}\right)+$ $\cdots+\left(u_{k-1}^{*}+u_{k}^{*}\right)=V_{1,2}+V_{3,4}+\cdots+V_{k-1, k}$ and $\sum_{i=1}^{k} u_{i}^{*}=\left(u_{2}^{*}+u_{3}^{*}\right)+\left(u_{4}^{*}+u_{5}^{*}\right)+\cdots+\left(u_{k}^{*}+u_{1}^{*}\right)=$ $V_{2,3}+V_{4,5}+\cdots+V_{k, 1}$. Thus, $V_{1,2}+V_{3,4}+\cdots+V_{k-1, k}=V_{2,3}+V_{4,5}+\cdots+V_{k, 1}$. This is a contradiction to the assumption that $V$ is generic.

\section{Manea's Model}

A special case of our model has been studied by Manea [2011]. Here, given an undirected network $G$ with the set of links denoted by $E$, the feasible coalitions are the pairs $(i, j) \in E$. The value of each coalition is one unit. To analyze this game, Manea introduces several new concepts, such as mutually estranged sets, partners and shortage ratios. Using our

\footnotetext{
${ }^{21}$ For example, by choosing $w_{i}$ to be 0 and $z_{i j}=V_{i j}$, we get a finite upper bound.
} 
convex program approach, some of the equilibrium computation in Manea [2011] can be greatly simplified. In particular, consider the following convex program that characterizes the stationary equilibrium of Manea's model.

$$
\begin{aligned}
\text { minimize: } & \sum_{i} u_{i}^{2} \\
\text { subject to: } & u_{i}+u_{j} \geq 1 \quad \forall(i, j) \in E .
\end{aligned}
$$

By Lemma 3.1, a feasible $u^{*}$ is the optimal solution of (15) if and only if there exists $\lambda_{i j} \geq 0$ for all $(i, j) \in E$ such that

$$
\begin{aligned}
& u_{i}^{*}=\sum_{j} \lambda_{i j}, \\
& \lambda_{i j}\left(u_{i}^{*}+u_{j}^{*}-1\right)=0 .
\end{aligned}
$$

Let $G^{*}$ be the subnetwork of $G$ that consists of the links $(i, j)$ for which $u_{i}^{*}+u_{j}^{*}=1$. Parts of the results in Theorem 3 of Manea [2011] can be stated as follows.

PROPOSITION C.1 Let $M$ be an independent set in $G^{*}$, let $L$ be the set of partners of $M$, i.e, the set of nodes that is connected to $M$ by a link in $G^{*}$, then

$$
\min _{i \in M} u_{i}^{*} \leq \frac{|L|}{|L|+|M|} \text { and } \max _{j \in L} u_{j}^{*} \geq \frac{|M|}{|L|+|M|}
$$

Proof. Proof: Because $M$ is an independent set in $G^{*}, u_{i_{1}}^{*}+u_{i_{2}}^{*}<1$ for $i_{1}, i_{2} \in M$. Because of (16) $\lambda_{i_{1} i_{2}}=0$, and thus,

$$
\sum_{i \in M} u_{i}^{*}=\sum_{i j: i \in M, j \in L} \lambda_{i j} \leq \sum_{j \in L} u_{j}^{*} .
$$

The last inequality holds because nodes in $L$ might have partners outside $M$. Now, because for every $i \in M$, there exists $j \in L$ such that $u_{i}^{*}+u_{j}^{*}=1$, and vice versa, thus,

$$
\min _{i \in M} u_{i}^{*}=1-\max _{j \in L} u_{j}^{*}
$$

Let $a=\min _{i \in M} u_{i}^{*}$, we have

$$
|M| \cdot a \leq \sum_{i \in M} u_{i}^{*} \leq \sum_{j \in L} u_{j}^{*} \leq|L| \cdot(1-a) .
$$

Therefore, $a \leq \frac{|L|}{|L|+|M|}$ and $1-a \geq \frac{|M|}{|L|+|M|}$. 
Parts of the results in Theorem 4 and 5 of Manea [2011] can be stated as follows.

PROPOSITION C.2 Let $H$ be a connected component of $G^{*}$, then one of the following is true.

- $H$ is a bipartite graph with two classes $L, M$, and

$$
u_{i}^{*}=\frac{|L|}{|L|+|M|} \text { for } i \in M \text { and } u_{j}^{*}=\frac{|M|}{|L|+|M|} \text { for } j \in L \text {. }
$$

- $H$ is non bipartite and $u_{i}^{*}=1 / 2$ for all $i \in H$, furthermore, $H$ can be covered by $a$ disjoint union of odd length cycles and matchings.
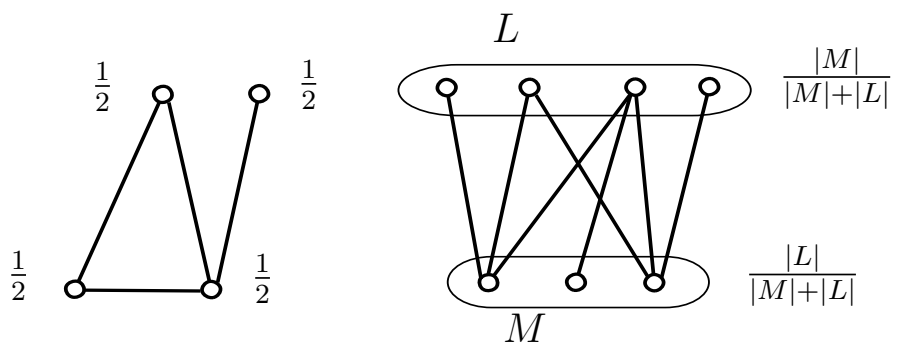

Figure 5: A payoff characterization

\section{Proof. Proof:}

First, consider the case when $H$ is a bipartite graph with two sides $L$ and $M$. See Figure 5 for a graphical representation. Because $H$ is a connected component of $G^{*}$, there exists $a>0$ such that $u_{i}^{*}=a$ for all $i \in M$, and $u_{j}^{*}=1-a$ for all $j \in L$.

Because there are no links in $G^{*}$ connecting $H$ and $G-H$, we have $u_{k}^{*}+u_{l}^{*}>1$ for $k \in H, l \in G-H$. Therefore, there exists $\epsilon>0$ such that by increasing (decreasing) all $u_{i}^{*}, i \in M$ by $\epsilon$ and decreasing (increasing) all $u_{j}^{*}, i \in L$ by $\epsilon$, the new solution does not violate any constraint of (15).

Now, $u_{i}^{*}=a$ for all $i \in M$ and $u_{j}^{*}=1-a, j \in L$ is in the optimal solution of (15). Consider the value

$$
\sum_{i \in M} u_{i}^{* 2}+\sum_{j \in L} u_{j}^{* 2}=|M| a^{2}+|L|(1-a)^{2}
$$


Because we can either increase or decrease $a$ by $\epsilon>0$, the value above is optimal if the derivative of $|M| a^{2}+|L|(1-a)^{2}$ is zero. This gives $a=\frac{|L|}{|L|+|M|}$.

Second, if $H$ is a non-bipartite graph, then consider an odd length cycle of $H$. Because $u_{i}^{*}+u_{j}^{*}=1$ holds for every link $i, j$ along the odd length cycle, we have $u_{i}^{*}=1 / 2$ for all $i \in H$.

Now because of (16), there exists $\lambda_{i j} \geq 0$ for all links $i j$ in $H$ such that

$$
\frac{1}{2}=\sum_{j \in H} \lambda_{i j} \text { for all } i \in H
$$

Prior research (see, for example, Lovász and Plummer [1986]) has shown that this condition is equivalent to the fact that $\mathrm{H}$ can be covered by odd cycles and matching. 